\title{
LA-UR-13-20621
}

Approved for public release; distribution is unlimited.

Title:

Author(s):

Intended for:

\section{Subcritical Alpha Measurements}

Rawool-Sullivan, Mohini

Hayes, David K.

Briefing to US GOV agencies

\section{- LosAlamos

\author{
EST. 1943
}

Disclaimer:

Los Alamos National Laboratory, an affirmative action/equal opportunity employer,is operated by the Los Alamos National

Security, LLC for the National NuclearSecurity Administration of the U.S. Department of Energy under contract DE-AC52-06NA25396.

By approving this article, the publisher recognizes that the U.S. Government retains nonexclusive, royalty-free license to

publish or reproduce the published form of this contribution, or to allow others to do so, for U.S. Government purposes.

Los Alamos National Laboratory requests that the publisher identify this article as work performed under the auspices of the

U.S. Departmentof Energy. Los Alamos National Laboratory strongly supports academic freedom and a researcher's right to publish; as an institution, however, the Laboratory does not endorse the viewpoint of a publication or guarantee its technical correctness. 


\title{
Subcritical Alpha Measurements
}

\author{
Mohini Rawool-Sullivan (IAT-1) \\ David Hayes (NEN-2) \\ January 30, 2013
}

- Los Alamos 


\section{Fission Chain}

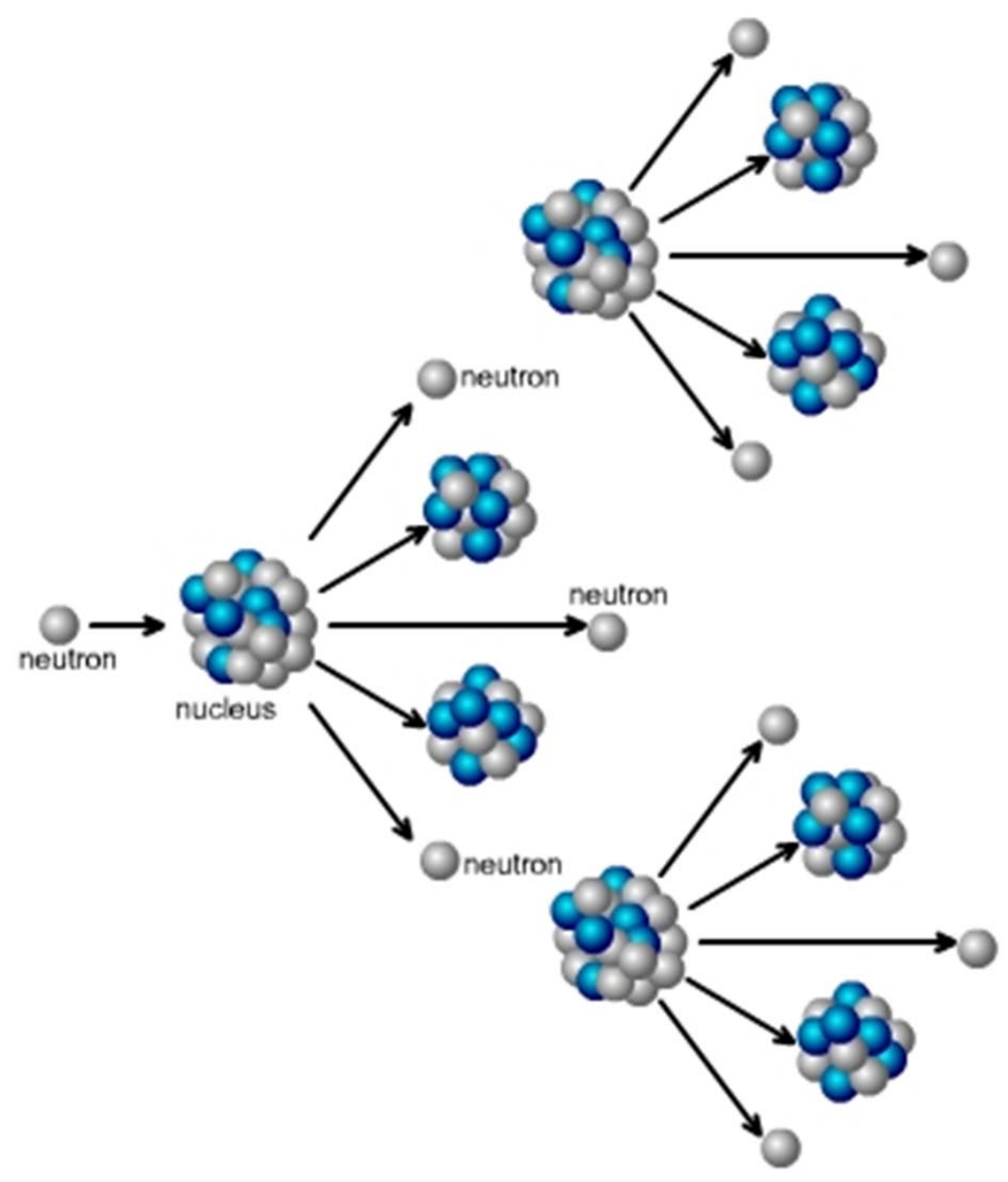

- In a reactor one neutron from a fission to induce another fission

- Statistical process due to very large number of neutrons and fission chains at one time

- Other neutrons are absorbed (and don't cause a fission) or leak out of the reactor

- A reactor is critical when it maintains a steady-state neutron chain - the total number of neutrons (power) in the reactor is constant

- If a reactor is subcritical, the total number of neutrons (power) decreases

- If a reactor is supercritical, the total number of neutrons (power) increases 


\section{Multiplication Factor}

- $\mathbf{k}_{\text {eff }}$ is the multiplication factor

- $k_{\text {eff }}$ is a measure of the number of neutrons in one generation compared to the previous generation

$$
k=\frac{\text { Number of neutrons in one generation }}{\text { Number of neutrons in preceeding generation }}
$$

- Three possible values for $k_{\text {eff }}$ :

- $k_{\text {eff }}<1$, neutron population is dying away, reactor is subcritical

- $k_{\text {eff }}=1$, neutron population is steady, reactor is critical

- $k_{\text {eff }}>1$, neutron population is increasing, reactor is supercritical 


\section{Neutron Multiplication}

- Neutron Multiplication is the total number of neutrons that would be generated through fission from a single starter neutron

- Not the same as the multiplication factor $k_{\text {eff }}$ !

- Only for subcritical reactors

$$
M=\frac{1}{1-k_{e f f}}
$$

Example: $\mathrm{k}_{\mathrm{eff}}=\mathbf{0 . 5}$

$1^{\text {st }}$ Generation: 1000

$2^{\text {nd }}$ Generation: 500

$3^{\text {rd }}$ Generation: 250

$4^{\text {th }}$ Generation: 125

Etc.

Total Neutrons: 2000

Total Neutrons $=\mathbf{M} *$ Starting Neutrons

$\mathbf{M}=\mathbf{2}$

\begin{tabular}{|c|c|}
\hline $\mathbf{k}_{\text {eff }}$ & $\mathrm{M}$ \\
\hline 0.5 & 2.0 \\
\hline 0.90 & 10.0 \\
\hline 0.95 & 20.0 \\
\hline 0.99 & 100.0 \\
\hline
\end{tabular}




\section{Region of Criticality}

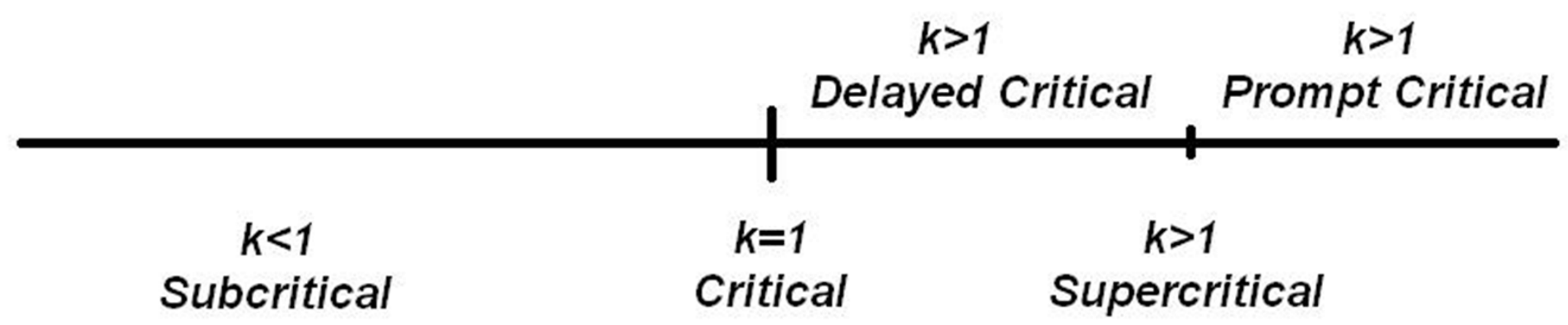

Neutrons produced in fission

Prompt neutrons: produced directly in fission event, $99 \%+$ of all fission neutrons

Delayed neutrons: from decay of fission fragments, $<1 \%$ of all fission neutrons

Delayed Neutron Fraction $=\beta=\frac{\text { Delayed neutrons }}{\text { Delayed neutrons }+ \text { Prompt neutrons }}$

Delayed Critical: Reactor needs both prompt and delayed neutrons to be critical

increase in neutron population (power) dominated by time for delayed neutrons to appear long time for power increase allows for control mechanisms

Prompt Critical: Reactor only needs prompt neutrons to be critical

increase in neutron population (power) dominated by time for prompt neutrons to appear short time for power increase does not allow for control mechanisms 


\section{Reactivity}

- Reactivity is a measure of how far from critical an assembly is, represented by $\rho$

- Related to the multiplication factor: $\rho=\frac{k_{e f f}-1}{k_{e f f}}$

- Three possible values for :

$-\quad \rho<0$, which means $k_{\text {eff }}<1$, so the reactor is subcritical

- $\rho=0$, which means $k_{\text {eff }}=1$, so the reactor is critical

- $\rho>0$, which means $k_{\text {eff }}>1$, so the reactor is supercritical 


\section{Units of Reactivity}

- Reactivity itself is unitless

- However, it can be quoted in units of:

- Dollars and cents

- pcm (per cent millirho)

- 1 dollar of reactivity $=\$ 1=\beta$

- 1 cent of reactivity $=\$ \mathbf{\$ 0} .01=\beta / 100$

- By definition, $\$ 1$ of reactivity is prompt critical

- Regions of supercriticality

- Delayed critical:

$0<\rho<\beta$

- Prompt Critical

$-\beta=\rho$

- Prompt supercritical

$\beta<\rho$ 


\section{"Measuring" Reactivity}

- Reactivity $\rho$ (and multiplication factor $k_{\text {eff }}$ ) cannot be directly measured

- Reactivity can be inferred from other measurements

- Neutron leakage out of the reactor is proportional to the number of neutrons inside the reactor

- Larger the neutron leakage, the larger the neutron population

- Neutron detectors can be used to determine the neutron leakage, giving an idea of what the neutron population is

- Power increases lead to more neutrons, which lead to greater leakage

- Measurements of how power (i.e., leakage) increases or decreases over time can give an indication of reactivity of the system 


\section{Point Reactor Kinetics Model}

- The point kinetic equation for reactor (without an external source)

$\frac{d n(t)}{d t}=\frac{(\rho-\beta)}{\Lambda} n(t)+\sum_{i=1}^{6} \lambda_{i} c_{i} ;$

$n(t)$ represents the neutron population; $\lambda_{i} c_{i}$ precursors decaying by emitting a delayed neutron per second; $\beta$ is the delayed neutron group fraction and $\rho$ is the reactivity and $\Lambda$ represents the mean neutron generation time.

- Assuming that delayed neutrons can be neglected then equation takes a form $\frac{\partial n(t)}{\partial t}=\alpha n(t)$; with general solution $n(t)=n\left(t_{0}\right) e^{\int_{t_{0}}^{t} \alpha t}$ and $\alpha=\frac{\rho-\beta}{\Lambda}=\frac{1}{\mathrm{n}(\mathrm{t})} \frac{\partial n(t)}{\partial t}=\frac{d}{d t} \ln (n(t)) ;$

- $\alpha$ has units of 1 over time 


\section{$\alpha$ and $k_{\text {eff }}$ for prompt critical reactor}

- The average neutron lifetime $l$ is the characteristic multiplication time or generation time for prompt critical

- If there are $n(t)$ neutrons in the system at a give tine, $t$, at a time, $l$, later there will be $n(t+l)$ neutrons. The magnitude of the $n(t+l)$ will depend on prompt critical multiplication factor $k_{p}: n(t+l)=$ $n(t) k_{p} \approx n(t)+l \frac{\partial n(t)}{\partial t}$

- Rearranging above equation $\frac{\partial n(t)}{\partial t}=\frac{k_{p}-1}{l} n(t)$

- The general solution is then $n(t)=n\left(t_{0}\right) e^{\int_{t_{0}}^{t} \frac{k p-1}{l} t}=n\left(t_{0}\right) e^{\int_{t_{0}}^{t} \alpha t}$

$$
\alpha(t)=\frac{k_{p}-1}{l}=\frac{1}{n(t)} \frac{\partial n(t)}{\partial t}=\frac{d \ln (n(t))}{d t}
$$




\section{$\alpha$ and Criticality}

- If $\alpha$ is positive ( $\rho>\beta)$, then the core is super-promptcritical.

- If $\alpha$ is negative $(\rho<\beta)$, then the core is sub-promptcritical.

- If $\alpha$ is exactly zero $(\rho=\beta)$, then the reactor is prompt critical. 


\section{Measuring $\alpha$ for Supercritical System}

- Absolute leakage neutron or gamma flux can be measured as long as the calibration of detection system is detailed enough to produce an accurate absolute flux measurement.

- By calculating the derivative of the natural log of the measured gamma flux, the function obtained $\alpha$ is free from the timedependent amplitude constants (and their uncertainties) required for an absolute flux measurement. (LA-UR-10-04510). This method can also be applied to neutron flux.

- Using equation $\alpha=\frac{k_{p}-1}{l}$ value of $k_{p}$ can be obtained.

- The reactivity $\rho=\frac{k_{p}-1}{k_{p}}$ 


\section{Neutron Detector Pulse Trains}

(a)

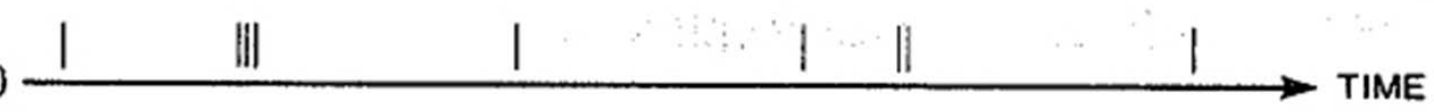

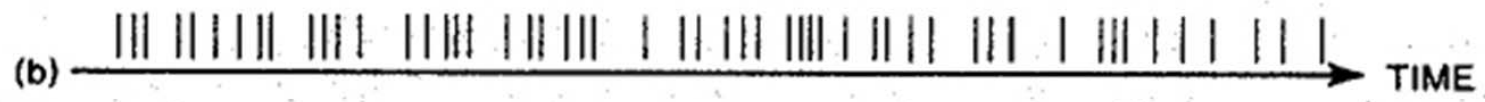

Fig. 16.I Neutron pulse trains as they might appear on a time axis. (a) An idealized pulse train containing correlated and uncorrelated events. (b) An actual pulse train observed at high counting rates using a detector with typical efficiency and die-away-time characteristics.

"Principles of neutron coincidence counting," N. Ensslin, Chapter 16,

Passive Nondestructive Assay Manual - PANDA, Ed. Doug Reilly, N. Ensslin and Hastings Smith Jr. NUREG/CR-5550, LA-UR-90-732, NRC FIN A7241 .

http://www.lanl.gov/orgs/n/n1/panda/index.shtml 


\section{Measuring $\alpha$ for Subcritical Assemblies : Rossi-Alpha Distribution}

"Principles of neutron coincidence counting," N. Ensslin, Chapter 16, Passive Nondestructive Assay Manual - PANDA, Ed. Doug Reilly, N. Ensslin and Hastings Smith Jr. NUREG/CR-5550, LAUR-90-732, NRC FIN A7241

- This Rossi-alpha distribution is obtained by starting a clock at $t=0$ with the arrival of an arbitrary pulse. The clock continues to run, and each succeeding pulse is stored by a mutiscaling circuit in a bin corresponding to its arrival time. A typical bin width might be $1 \mu \mathrm{s}$, and the total number of time bins available might range from 1024 to 4096 . When the end of the total time interval is reached, the clock is stopped and the circuit remains idle until another event restarts the process at $t=$ 0 again. Thus the Rossi-alpha distribution is the distribution in time of events that follow after an arbitrarily chosen starting event. If only random events are being detected, the distribution is constant with time. If real coincidence events are also present, the Rossi-alpha distribution is given by

$$
S(t)=A+R e^{-\alpha t} ; \mathrm{S}(\mathrm{t}) \text { is the height of the distribution at time } \mathrm{t} ; \mathrm{A} \text { is the }
$$

accidental, or random, count rate; $\mathrm{R}$ is the real coincidence count rate and $\alpha=\frac{1}{\tau}$; where $\tau$ is the detector die-away time. 


\section{The Rossi-Alpha Distribution}

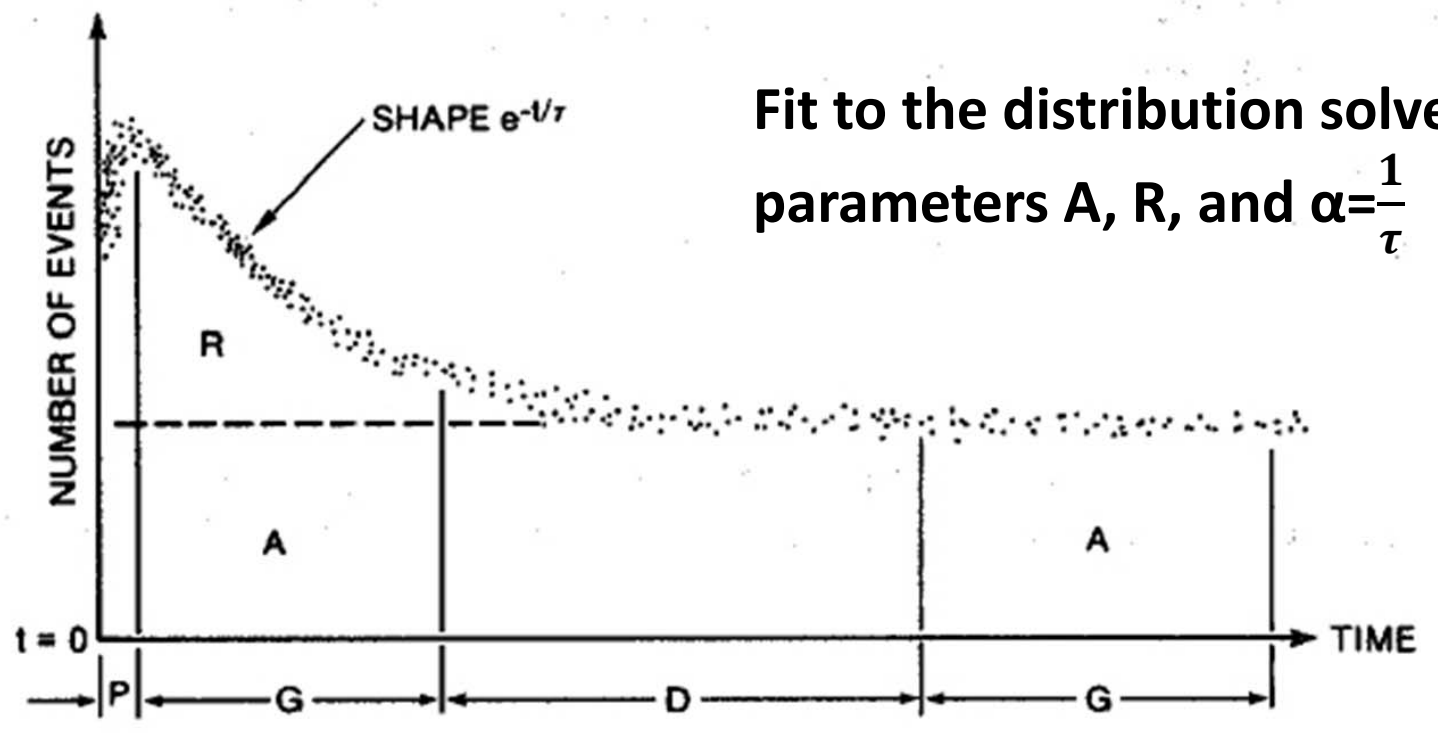

Fig. 16.3 A Rossi-alpha distribution showing detected neutron events as a function of time following an arbitrary starting event. $R$ represents real coincidence events, and $A$ represents accidental coincidence events. $P=$ predelay, $\boldsymbol{G}=$ prompt and delayed gates, $D=$ long delay, and $\tau=$ die-away time.

"Principles of neutron coincidence counting," N. Ensslin, Chapter 16, Passive Nondestructive Assay Manual - PANDA, Ed. Doug Reilly, N. Ensslin and Hastings Smith Jr. NUREG/CR-5550, LA-UR-90-732, NRC FIN A7241 


\section{Coincidence Circuits : some terminology}

- Coincidence circuits generally contain electronic components called "one-shots" or "gate generators" that produce an output pulse of fixed duration whenever an input pulse is received.

- Gate generators are used to convert the input pulses from the neutron detector into very short output pulses are called "triggers."

- Gate generators used to convert the input pulses into long output pulses are called "gates."

"Principles of neutron coincidence counting," N. Ensslin, Chapter 16, 


\section{Rossi-Alpha to Feynman Variance}

- Rossi-Alpha is useful for high-multiplication assemblies

- For lower multiplication assemblies Feynman variance method (Feynman, DE Hoffman, Serber) can be used. This method was first used on the "LOPO" or low power aqueous fuel reactor system. LOPO could have been described as self-sustaining critical assembly.

- For a neutron flux in which no neutrons are correlated with any other neutrons the probability of getting $\mathrm{n}$ number of neutrons detected in a gate follows the Poisson expectation. However two or more neutrons emitted in a single fission event are correlated in time by reason of being born in the same fission and will get detected in the same gate. Correlated sources are not Poisson distributed. This departure can be expressed as

$\mathrm{Y}=$ variance-to-mean ratio -1 . 


\section{Using Moments of the Neutron Counting Distribution}

A. Robba, E. Dowdy, H. Atwater, “Neutron Multiplication Measurements Using Moments of the Neutron Counting Distribution," Nuc. Instr. and Meth., 215, 473-479 (1983).

D. Cifarelli, W. Hage, "Models for a Three-Parameter Analysis of Neutron Signal Correlation Measurements for Fissile Material Assay," Nuc. Instr. Meth. A251, 550-563, 1986.

- Relates the measured count rates to the subcritical assembly properties based on a point model.

- The single events, double events and triple events are the measured quantities, the efficiency and gate fractions are characteristics of the detector used. The factorial moments of the spontaneous and induced fission distributions are fundamental nuclear physics values and are fixed quantities for an given assembly. The point model equations are solved to get multiplication, $\alpha$ and mass of the fissile material 


\section{Interval distribution}

- Perform frequency binning on "t2-t1", "t3-t2", etc...

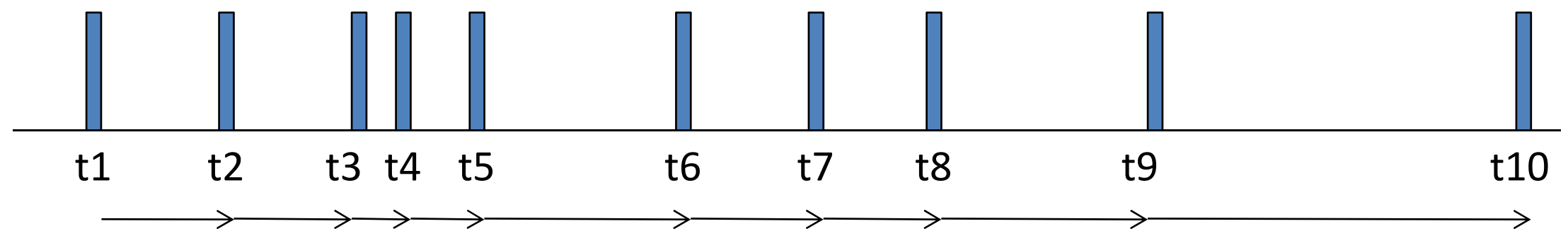

- The interval distribution is the distribution of time intervals between detected events.

- Applicable to randomly pulsed neutron source on the assembly 


\section{Interval distribution}

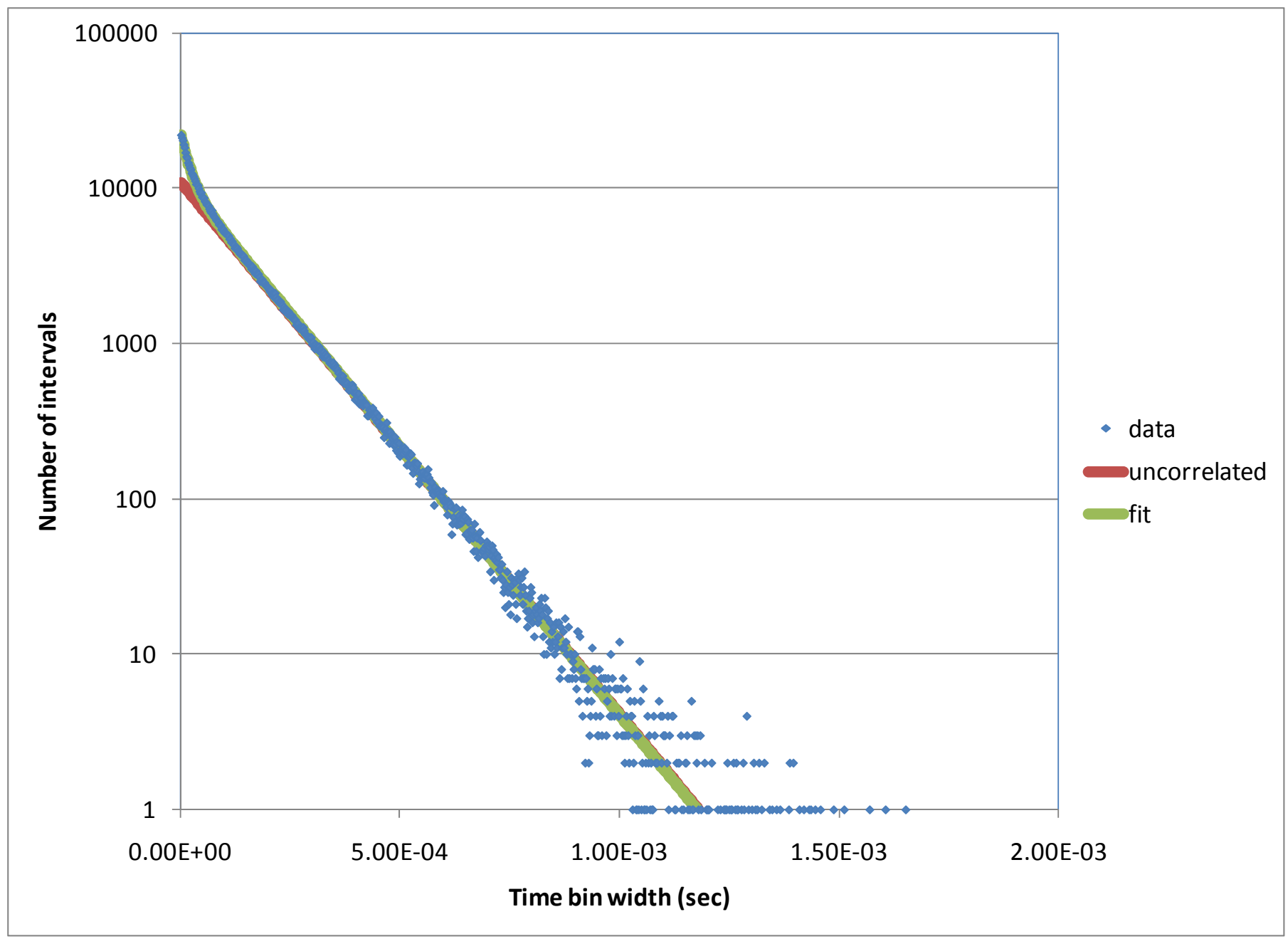

Hutchinson et. al., "Overview of Several Subcritical Measurement Methods," LA-UR-11-03807 - Los Alamos 


\section{Interval distribution}

- Parameters

$-\mathrm{s}$ is the starter neutron emission rate

$-\mathrm{C}$ is the detector count rate (cts/sec)

- Other terms same as previous definitions

- We use:

- Known parameters: $\mathrm{S}, \bar{v}$ and $D_{v}$

- Solve for: $\mathrm{k}_{\mathrm{p}}, \epsilon$, and $\Lambda$ (or $\ell$ ) 


\section{Interval distribution}

$$
\begin{aligned}
P_{C C}(\tau) & =k \exp \{-\sigma[(\gamma-1) \alpha \tau \\
& \left.\left.+2 \ln \frac{T}{4 \gamma}\right]\right\}\left(\frac{2 S}{T}\right)^{2}\left[1+\frac{2 \gamma^{2}}{\sigma S^{2}} \exp (-\alpha \gamma \tau)\right] \\
S= & (\gamma+1)+(\gamma-1) \exp (-\alpha \gamma \tau) \\
T & =(\gamma+1)^{2}+(\gamma-1)^{2} \exp (-\alpha \gamma \tau)
\end{aligned}
$$

- Fit to find $k, \alpha, \sigma$, and $\gamma$.

$$
\sigma=\frac{s \Lambda}{\bar{v} D_{v}}
$$

$$
\gamma=\sqrt{\left(1+\frac{2 \varepsilon D_{v}}{\rho_{p}^{2}}\right)}
$$$$
C=\frac{\sigma \alpha}{2}\left(\gamma^{2}-1\right)
$$

Hutchinson et. al., "Overview of Several Subcritical Measurement Methods," LA-UR-11-03807 


\section{The Cf-252 source-driven noise analysis (CSDNA) method}

- Relates measured quantities to the multiplication of the system.

- Requires use of $\mathbf{2}$ or more detectors for frequency domain measurement and (single detector for time domain measurement) a ${ }^{252} \mathrm{Cf}$ source inside an ionization chamber. ${ }^{252} \mathrm{Cf}$ is deposited on a electrode thus each spontaneous fission of the source creates a pulse defining time of the ${ }^{252} \mathrm{Cf}$ fission.

- Simultaneous Rossi-Alpha and Randomly pulsed neutron source measurement

- Note that this is a semi-empirical method, requiring an eigenvalue $\left(k_{\text {eff }}\right)$ calculation 


\section{Use of gamma detectors}

- In principle all these measurements could be made using gamma detectors

- Gamma emitted in fission are directly proportional to number of fissions. However in low-multiplication systems it is difficult to infer rate of change in gamma flux.

- For subcritical assemblies neutron measurements are simpler and frequently used 


\section{Neutron Detectors Used}

- Gas filled detectors:

$-\mathrm{BF}_{3},{ }^{3} \mathrm{He}$ for thermal neutrons

$-{ }^{4} \mathrm{He}, \mathrm{CH}_{4}$ for fast neutron detectors

- Plastic Scintillators

- Liquid scintillators

- Long Counters

For long counter see "Total Neutron Counting Instruments and Applications," J. Sprinkle, Chapter 15, Passive Nondestructive Assay Manual - PANDA, Ed. Doug Reilly, N. Ensslin and Hastings Smith Jr., NUREG/CR-5550, LA-UR-90-732, NRC FIN A7241.

http://www.lanl.gov/orgs/n/n1/panda/00326410.pdf 


\section{SNAP detector}

- The Shielded Neutron Assay Probe (SNAP) for total neutron counting - Verify Pu-240 content of the metal

- SNAP detectors consists of one or two ${ }^{3} \mathrm{He}$ counters embedded in a polyethylene cylinder. The polyethylene cylinder is wrapped in a thin cadmium sheet to preferentially absorb any background of thermal neutrons. Cadmium also prevents thermal neutrons from scattering back from the SNAP to the object. 


\section{Neutron Pods (NPOD3)}

- 15 10-atm ${ }^{3} \mathrm{He}$ tubes in two rows moderated by polyethylene

- Records Data in a list mode format

- Records the tube a neutron is absorbed in and the time the absorption occurs. This allows for an analysis of the data with various analysis methods described before and an archive of the data for later analysis.
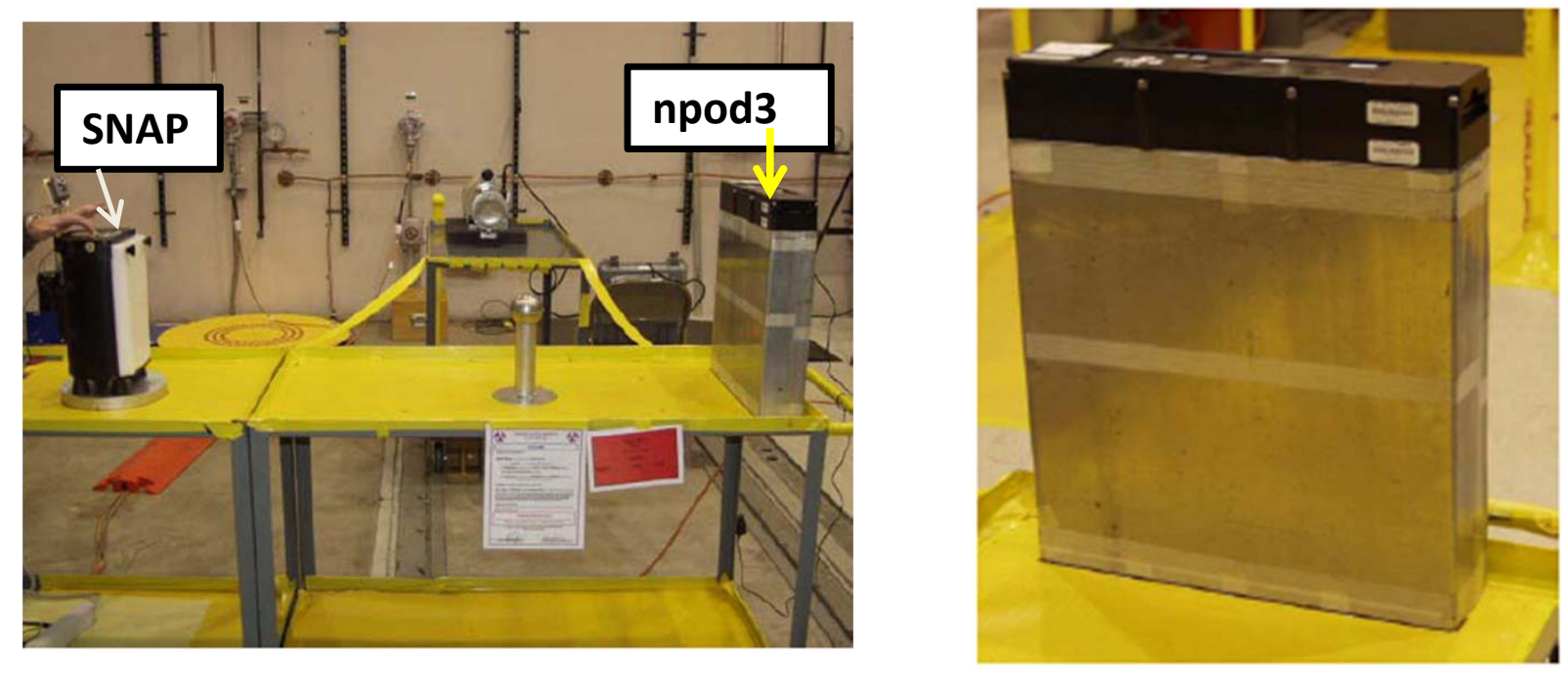

Solomon Clell J. et. al., "Covariance-Informed Energy-Dependent Perturbations to the Pu-239 nu-bar with Subcritical Polyethylene-Reflected Pu NPOD Data," LA-UR-12-25879, MCNP/ENDF/NJOY Workshop, 2012-10-30 


\section{Summary}

- This presentation introduces basic concepts of reactor dynamics. Additionally criticality parameters and their measurements for subcritical assemblies are discussed. 